\title{
The indentation response of Nickel nano double gyroid lattices
}

\author{
S.N. Khaderi ${ }^{\text {a,b }}$, M.R.J. Scherer ${ }^{\text {c }}$, C.E. Hall ${ }^{\text {a }}$, U. Steiner ${ }^{\text {d }}$, U. Ramamurty ${ }^{\mathrm{e}}$, \\ N.A. Fleck ${ }^{\mathrm{a}}$ and V.S. Deshpande ${ }^{\mathrm{a}^{*}}$ \\ ${ }^{a}$ Department of Engineering, University of Cambridge, \\ Trumpington Street, Cambridge CB2 1PZ, UK. \\ ${ }^{\mathrm{b}}$ Department of Mechanical and Aerospace Engineering, Indian Institute of Technology \\ Hyderabad, Yeddumailaram - 502 205, Telangana, India. \\ ${ }^{c}$ Papierfabrik Louisenthal GmbH, 83701 Gmund am Tegernsee, Germany. \\ ${ }^{\mathrm{d}}$ Adolphe Merkle Institute, Chemin des Verdiers 4, CH-1700 Fribourg, Switzerland. \\ ${ }^{\mathrm{e}}$ Department of Materials Engineering, Indian Institute of Science, \\ Bangalore-560 012, India.
}

\begin{abstract}
The indentation response of Nickel nano double gyroid films has been measured using a Berkovich nano indenter and the effective mechanical properties of the $\mathrm{Ni}$ double gyroid lattices inferred via a multi-scale finite element analysis. The $1 \mu \mathrm{m}$ thick double gyroid films were manufactured by block copolymer self-assembly followed by electrodeposition of the $\mathrm{Ni}$ resulting in two interpenetrating single gyroids of opposite chirality, an overall relative density of $38 \%$ and a cell size of about $45 \mathrm{~nm}$. The measured hardness was $\sim 0.6 \mathrm{GPa}$ with no discernable indentation size effect. A multi-scale finite element (FE) analysis revealed that the uniaxial compressive strength is approximately equal to the hardness for this compressible lattice. Thus, the $38 \%$ relative density $\mathrm{Ni}$ double gyroid has a strength equal to or greater than the strongest fully dense bulk Ni alloys. The FE calculations revealed that this was a consequence of that fact that the $\mathrm{Ni}$ in the $13 \mathrm{~nm}$ gyroid struts was essentially dislocation free and had a strength of about $5.7 \mathrm{GPa}$, i.e. approaching the theoretical strength value of $\mathrm{Ni}$. The measurements and calculations reported here suggest that inspite of the nano gyroids having a bending-dominated topology they attain strengths higher than those reported for stretching-dominated micron scale lattice materials made via 3D printing. We thus argue that relatively fast and easy selfassembly processes are a competitive alternative to $3 \mathrm{D}$ printing manufacture methods for making high strength lattice materials.
\end{abstract}

Keywords: Lattice materials, nano-lattices; size effects; indentation.

* Corresponding author. E-mail address: vsd@eng.cam.ac.uk . 


\section{Introduction}

Over the past decade there has been considerable research into strategies to develop materials with the aim of filling holes in material property space as defined in Ashby charts [1]. One approach to filling these gaps in material property space is that of manipulating chemistry, developing new metal alloys, new polymer formulations and new compositions of glass and ceramics. A second is that of manipulating microstructure, using thermo-mechanical processing to control the distribution of phases and defects within materials. Both have been exploited systematically, leaving little room for further gains, which tend to be incremental rather than step-like. A third approach is that of controlling architecture to create hybrid materials combinations of materials or of material and space in configurations that offer enhanced performance. The success of carbon and glass-fibre reinforced composites at one extreme, and of foamed materials at another in filling previously empty areas of the strength density property space as seen in Fig. 1 has resulted in intense recent activity in the so-called lattice materials [2].

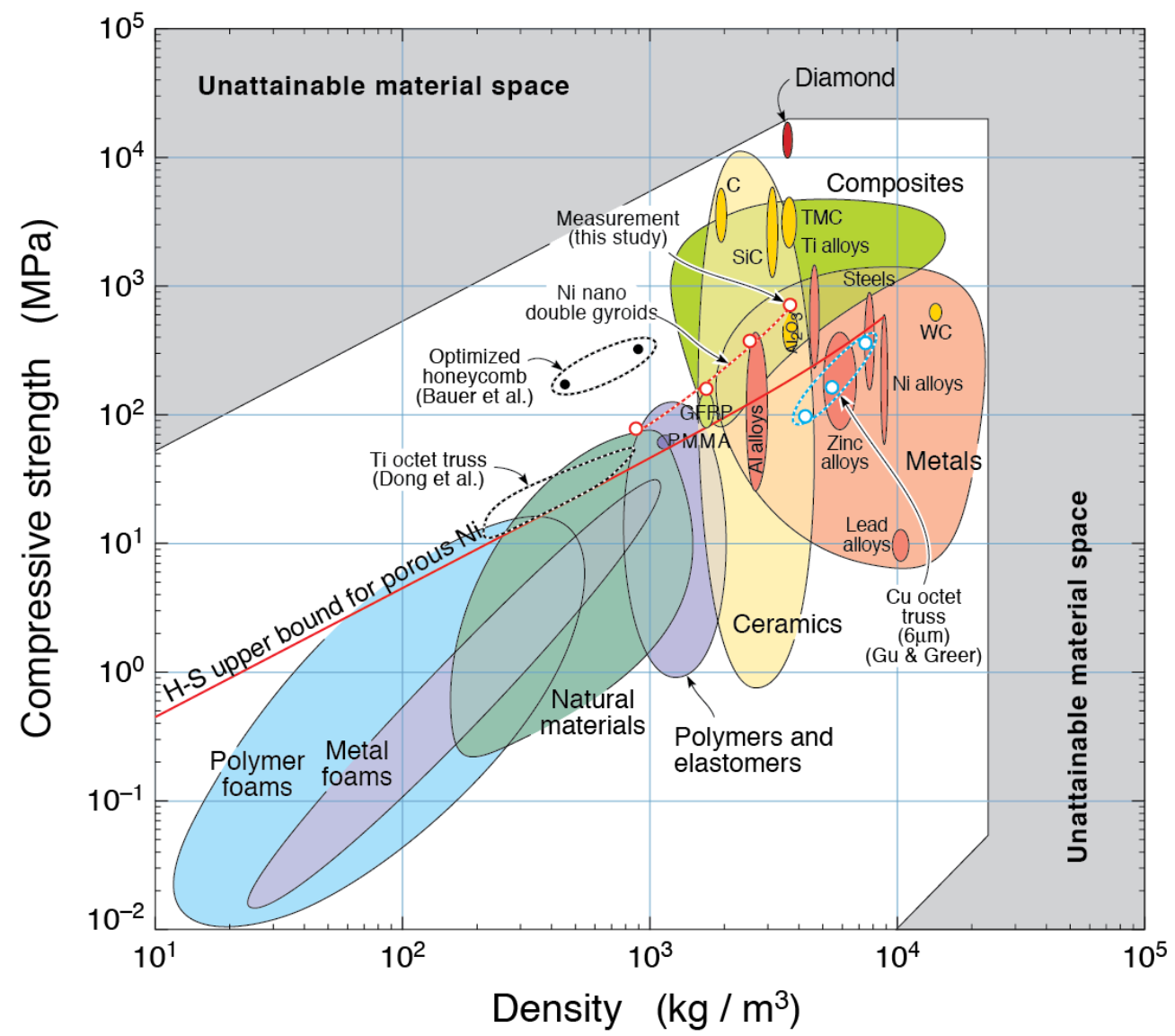

Figure 1: A strength versus density Ashby chart showing position of the Ni nano double gyroid lattices in strength-density space. The measured properties of macro Ti octet truss lattices (Dong et al. [14]), Cu micro octet truss lattices (Gu and Greer [9]) and micro ceramic honeycombs (Bauer et al. [12]) are highlighted.

Lattice materials are cellular, reticulated, truss or lattice structures made up of a large number of uniform lattice elements (e.g. slender beams or rods) and generated by tessellating a unit cell, comprised of just a few lattice elements, throughout space. Spatial or 3D lattices can be generated by filling space from polyhedral to generate effective solids with a volume fraction of solid material $\bar{\rho}$ (referred to subsequently as 
relative density). Of the regular polyhedra with a small number of faces only the cube and the rhombic dodecahedra can be tessellated to fill all space [3]. Typically spatial lattices are constructed using combinations of different polyhedral. For example, tetrahedra and octahedra may be packed to form the octet truss lattice [4]. There are two distinct species of lattice materials in terms of their mechanical properties. The first, typified by foams, are bending-dominated structures whose strength scales with $\bar{\rho}^{3 / 2}$ and the second, are stretching-dominated with strength scaling linearly with $\bar{\rho}$. To give an idea of the difference, a bending-dominated foam with $\bar{\rho}=0.1$ is less strong by a factor of 3 than a stretching-dominated octet truss lattice of the same relative density. The macroscopic properties are largely dictated by the connectivity of joints rather than by the regularity of the microstructure as discussed by Deshpande et al. [5].

Significant advances in the design and manufacture of lattice materials of various topologies with the aim of filling gaps in strength-density property space have been reported over the past few years driven largely (but not exclusively) by the development of 3D printing technology. Most of these lattice materials have stretching-dominated topologies for the reasons described above and fall into two categories: (i) macro lattices defined as lattice materials with struts in the millimetre or larger length scale [6-8] and (ii) micro/nano lattice materials with features sizes on the micron or sub-micron length scale [9-12].

The potential of macro-lattices in filling gaps in property space is readily determined by employing "continuum" bounds on properties. For example the space in Fig. 1 labelled as "unattainable" is based on the fact that either no element heavier than $\sim 25 \mathrm{Mg} \mathrm{m}^{-3}$ is known or exist and at low densities the highest achievable strength is limited by the Voigt bound: a porous solid made from a parent material with yield strength $\sigma_{Y S}$ is limited to have a strength $\sigma_{Y}=\bar{\rho} \sigma_{Y S}$ at the relative density $\bar{\rho}$. Thus, the unattainable material space in the top half of Fig. 1 is based on the highest strength that can be achieved by lattice materials made from solid diamond. Highly anisotropic micro-structures such as laminates or fibre composites can attain the Voigt bound. The upper bound on the strength of an isotropic porous solid can be estimated using the non-linear Hashin-Shtrikman upper bound [13] given by

$$
\frac{\sigma_{Y}}{\sigma_{Y S}}=\frac{2 \bar{\rho}}{\sqrt{(1-\bar{\rho})+4\left[1+\frac{2}{3}(1-\bar{\rho})\right]}} .
$$

The optimal strength of macro lattices made from the strongest solid $\mathrm{Ni}$ alloy is indicated in Fig. 1 using Eq. (1.1). There are no known micro-structures of lattice materials that achieve this non-linear Hashin-Shtrikman upper bound but the octet truss lattice is known to have theoretical properties that are reasonably close. Dong et al. [14] reported in a comprehensive study on $\mathrm{Ti}$ octet truss lattices (compressive measurements included in Fig. 1) and these materials have the highest strength to density ratios of macro lattice materials reported to-date.

The push to design micro/nano lattices has been driven by the work on size effects in the strength of metals. This "smaller is stronger" effect has been observed when crystalline metals are subjected to either strain gradients with micron/submicron 
wavelengths $[15,16]$ or sub-micron size specimens subjected to uniaxial deformation $[17,18]$. The use of this material strength size effect has been previously been exploited in nanoporous foams: these foams have been shown to have strengths higher than their macro counterparts. Advances in micro/nano 3D printing technologies such 2-photon lithography [12,19,20] and various types of microstereolithography $[10,11]$ have enabled these ideas to be extended to manufacture architecture micro/nano lattices with stretching-governed topologies. In fact, $\mathrm{Gu}$ and Greer [9] have shown that $\mathrm{Cu}$ octet truss lattices with $\bar{\rho} \approx 0.5$ can have a strength higher than fully dense bulk $\mathrm{Cu}$. Thus, continuum bounds such as Eq. (1.1) with $\sigma_{Y S}$ estimated from bulk strength measurements of the solid material can significantly underestimate the potential of micro/nano lattices in filling gaps in strength-density property space.

Here we report the manufacture and mechanical properties of $\mathrm{Ni}$ gyroid lattice materials made via a self-assembly route. This manufacturing process not only has the advantage of being faster compared to most 3D printing technologies, it also permits the manufacture of gyroid lattices with strut dimensions on the order of $\sim 10 \mathrm{~nm}$ : current 3D printing routes are unable to achieve this resolution. Struts with $10 \mathrm{~nm}$ diameters are expected to fully exploit the "smaller is stronger" effect and the main aim of this study is to investigate this potential.

(a)

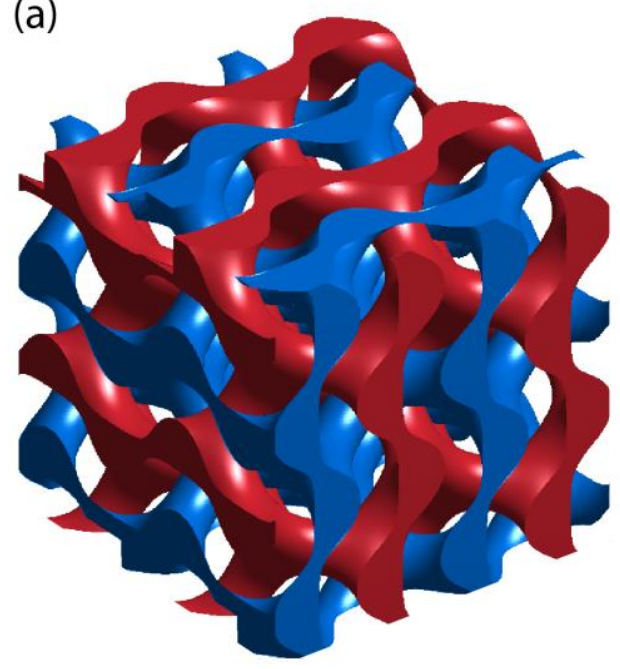

(b)

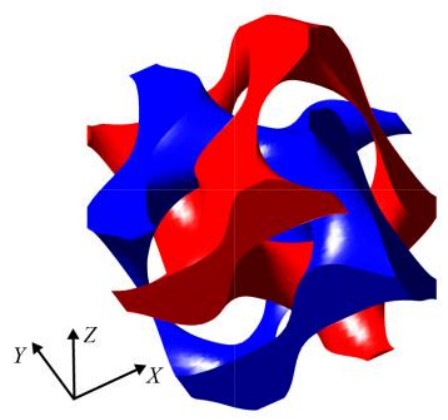

Figure 2: (a) Sketch of the double gyroid lattice comprising two interpenetrating single gyroids of opposite chirality. In this sketch a double gyroid with $2 \times 2 \times 2$ units is shown with each single gyroid having a relative density $\bar{\rho}=0.19$ such that the double gyroid has a relative density $\bar{\rho}^{D G}=0.38$. (b) Sketch of the double gyroid unit cell used in the FE computations of the effective properties.

\section{Experimental protocol and measurements}

The overall aims of the experimental program are to measure the indentation response of Nickel nano double gyroid lattices and use these measurements to extract both the effective properties of the gyroid lattices and those of the parent solid material. 


\subsection{Manufacture of nano double gyroid lattice coatings}

The Nickel double gyroid thin film coatings of thickness $\sim 1 \mu \mathrm{m}$ were manufactured by block co-polymer self-assembly onto a soda lime glass slide of thickness $2.2 \mathrm{~mm}$ coated by a $200-300 \mu \mathrm{m}$ thick layer of fluorine-doped tin oxide (FTO) as described in detail by Scherer et al. [21]. Here for the sake of completeness we briefly describe the salient steps.

(a)

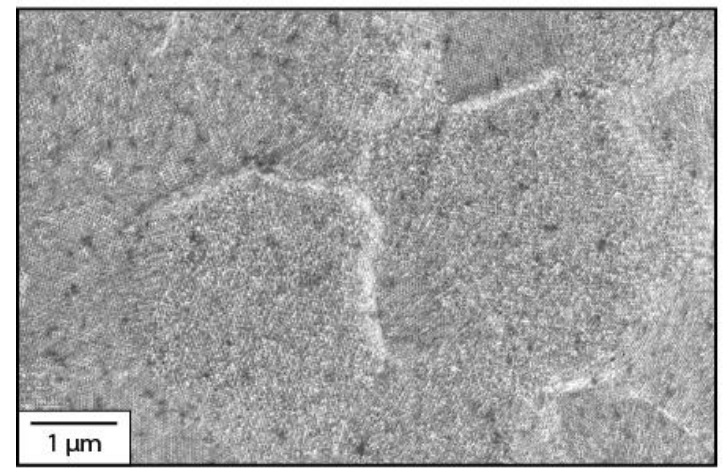

(b)

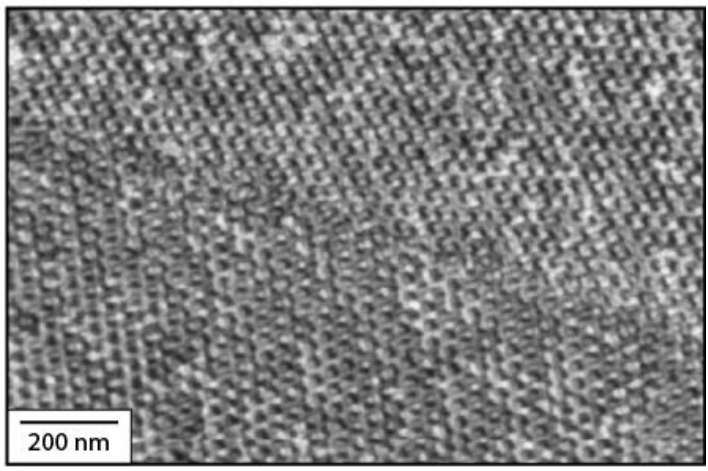

Figure 3: SEM micro-graphs of the top surface of the Ni double gyroid films in (a) low magnification showing the "grain" structure and (b) high magnification in which the $45 \mathrm{~nm}$ gyroid unit cells are visible.

First, the FTO coating was treated by a $0.1 \%$ solution of octyltrichlorosilane in anhydrous cyclohexane in order to make the coating slightly hydrophobic. Next, an approximately $1 \mu \mathrm{m}$ thick layer of poly(4-fluorostyrene-r-styrene)-b-poly(D,Llactide) (i.e. the copolymer) was spun coated on the FTO and the coated glass slide heated in a nitrogen atmosphere to $180^{\circ} \mathrm{C}$ in about 30 mins and then held at $205^{\circ} \mathrm{C}$ for 8 mins before cooling to $180^{\circ} \mathrm{C}$. The film was held at $180^{\circ} \mathrm{C}$ for $10 \mathrm{mins}$ before quenching to room temperature. This heat treatment results in the formation of the double gyroid topology of the lactide phase and the inverse double gyroid morphology of the polystyrene phase. The lactide is then dissolved using a $0.1 \mathrm{M}$ solution of sodium hydroxide (50:50 water to methanol) to leave behind the inverse polystyrene double gyroid. Nickel is then electro-deposited on the polystyrene lattice using Nickel Bright Finish solution (supplier Alfa Aesar) and finally the polystyrene dissolved with toluene to leave behind the Ni double gyroid lattice film on the FTO coating of the glass slide.

The co-polymer contains 37.9 vol\% lactide and hence this process produces a $\mathrm{Ni}$ double gyroid of relative density $\bar{\rho}^{D G}=0.38$ comprising two interpenetrating single gyroids as sketched in Fig. 2a. Each of these interpenetrating single gyroids have a relative density $\bar{\rho}=0.19$ with a unit cell size $\sim 45 \mathrm{~nm}$ and strut diameter of $\sim 13 \mathrm{~nm}$ as discussed in Scherer et al. [21]. Scanning electron micro-graphs (SEM) of the top surface of the gyroid films are shown in Figs. 3a and $3 \mathrm{~b}$ at two levels of magnification. The smaller magnification image in Fig. 3a clearly shows areas of different orientations of the gyroid lattices with "grain" boundaries separating these areas. Thus, these polycrystalline Ni double gyroid films have a columnar structure with in-plane grain sizes of $\sim 1.5 \mu \mathrm{m}$. The higher resolution image in Fig. $3 \mathrm{~b}$ gives some indication of the gyroid topology though even this resolution is insufficient to fully resolve the $45 \mathrm{~nm}$ unit cells. 
The thicknesses of the Ni double gyroid films of all specimens tested in this study were measured using a Zygo NewView 3D interferometer (i.e. the height difference between the FTO and gyroid film surfaces). All films had a thickness $h=0.9 \pm$ $0.1 \mu \mathrm{m}$ consistent with the $1 \mu \mathrm{m}$ target film thickness. In addition surface roughness measurements were also conducted using raster scanning by an indenter tip using a Hysitron Ub1 Nanoindenter system. An example of such a raster scan is included in Fig. 4. All the Ni double gyroid films tested in this study had an average roughness $R_{a} \approx 20 \mathrm{~nm}$.

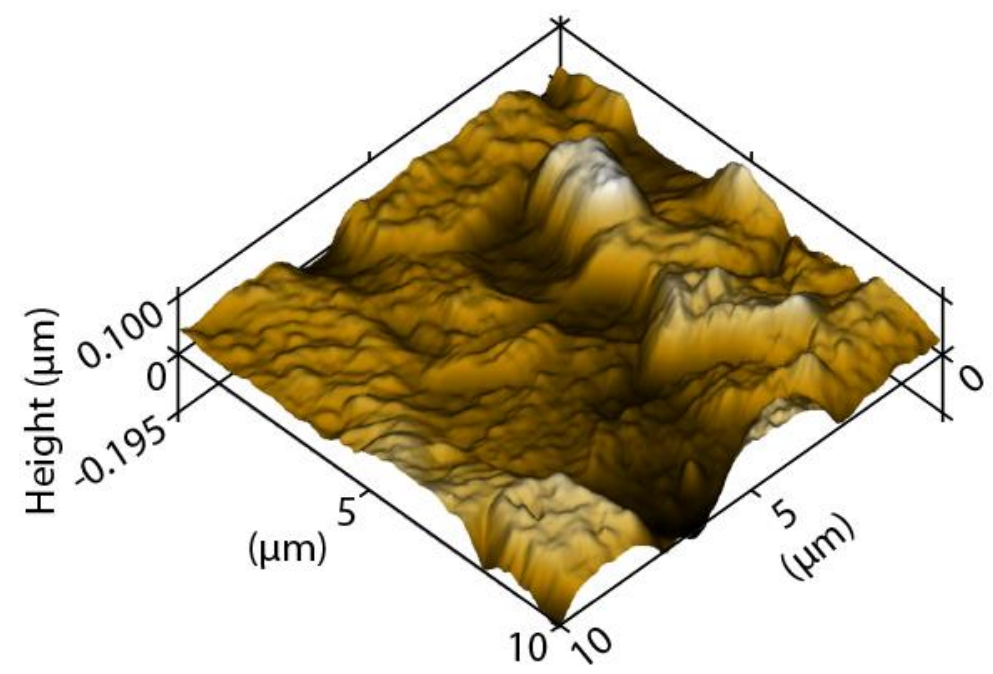

Figure 4: A raster scan of a $10 \mu \mathrm{m} \times 10 \mu \mathrm{m}$ patch of the surface of the Ni double gyroid film using the Nanoindenter system. The mean surface roughness is $R_{a} \approx 20 \mathrm{~nm}$.

\section{$2.1 \quad$ Indentation measurement protocol}

Berkovich nano indentation tests on $4 \mathrm{Ni}$ double gyroid films were performed using Hysitron Ub1 Nanoindenter system with a maximum indentation force of $10 \mathrm{mN}$ and a depth resolution of $0.04 \mathrm{~nm}$. The Berkovich tip had a tip radius of $50 \mathrm{~nm}$ and the usual Berkovich half angle of $65.35^{\circ}$ measured from the axis to one of the pyramidal flats. The calibration between the nominal contact area $A_{c}$ and the indentation depth $\delta$ as given by manufacturer (and confirmed by conducting indentation tests on fused silica glass samples with a modulus $69.6 \mathrm{GPa}$ ) is

$$
A_{c} \approx 24.5 \delta^{2}+0.7698 \delta
$$

where $A_{c}$ and $\delta$ are in $\mu \mathrm{m}^{2}$ and $\mu \mathrm{m}$, respectively. The tip is thus sufficiently sharp to be considered to be pyramidal at an indentation depth $\delta \geq 3 \mathrm{~nm}$.

Approximately six separate indentation tests were conducted on each of the 4 gyroid films. For each test 30 interrupted load/unload cycles were performed whereby the peak load in each cycle was progressively increased to the maximum machine load capacity of $10 \mathrm{mN}$ over the 30 cycles. Each loading and unloading segment was $2 \mathrm{~s}$ in duration with a $1 \mathrm{~s}$ hold between these segments. During each unloading cycle the load was reduced to $50 \%$ of the load just prior to unloading. The modulus and hardness were extracted from these measurements using the standard Oliver and 
Pharr [22] procedure. We note in passing that experiments were also performed by increasing the above loading times by a factor of 10: no appreciable change in the measurements was observed indicating that the responses measured here are reasonably strain rate insensitive. We outline this procedure here for completeness and to clarify the assumptions made in the context of compressible lattice films.

The hardness $H$ at an indentation depth $\delta$ is defined as $H(\delta) \equiv P / A_{t}$, where $P$ and $A_{t}$ are the applied indentation load and true projected contact area, respectively at the applied indentation depth $\delta$. While the nominal contact area $A_{c}$ is immediately known from $\delta$ via the tip area function (2.1), the true contact area $A_{t}$ is typically estimated in the indentation analysis of fully dense metals via correction factors to account for the effect of sink-in or pile-up around the indenter [22]. However, the double gyroid films are plastically compressible as discussed in Khaderi et al. [23] and finite element calculations presented in Section 3 confirm that there is negligible sink-in or pile-up around the indenter for these gyroid films. Thus, in the indentation analysis employed here we assume $A_{t}=A_{c}$ and the hardness $H$ follows directly from the measured load and indentation depth via the area tip function (2.1). The procedure to infer the Young's modulus $E^{D G}$ of the gyroid film is as follows. A power law curve of the form $P=c\left(\delta-\delta_{f}\right)^{m}$ is fitted to the measured unloading response $P(\delta)$ where $\delta_{f}, m$ and $c$ are constants to fit the measured curve. The unloading stiffness is then defined as $S \equiv$ $d P / d \delta$ at the peak load $P=P_{\max }$ at the indentation depth $\delta_{\max }$ just prior to unloading, i.e. $S \equiv m c\left(\delta_{\max }-\delta_{f}\right)^{m-1}$. The reduced Young's modulus $E_{R}$ of the double gyroid film and the indenter tip is then given via the Sneddon [24] formula as

$$
E_{R}=\frac{S}{2} \sqrt{\frac{\pi}{A_{t}}}
$$

where we will again assume $A_{t}=A_{c}$. With the reduced modulus now known the double gyroid film modulus $E^{D G}$ is inferred from $E_{R}$ using the usual contact relation

$$
\frac{1}{E_{R}}=\frac{\left[1-\left(v^{I}\right)^{2}\right]}{E^{I}}+\frac{\left[1-\left(v^{D G}\right)^{2}\right]}{E^{D G}}
$$

where $E^{I}=1140 \mathrm{GPa}$ and $v^{I}=0.07$ are the Young's modulus and Poisson's ratio, respectively of the diamond Berkovich indenter tip material [25] and $v^{D G}=0.35$ is the Poisson's ratio of the $\bar{\rho}^{D G}=0.38$ gyroid film; see Section 3 for the elastic properties of gyroid lattices. 
(a)

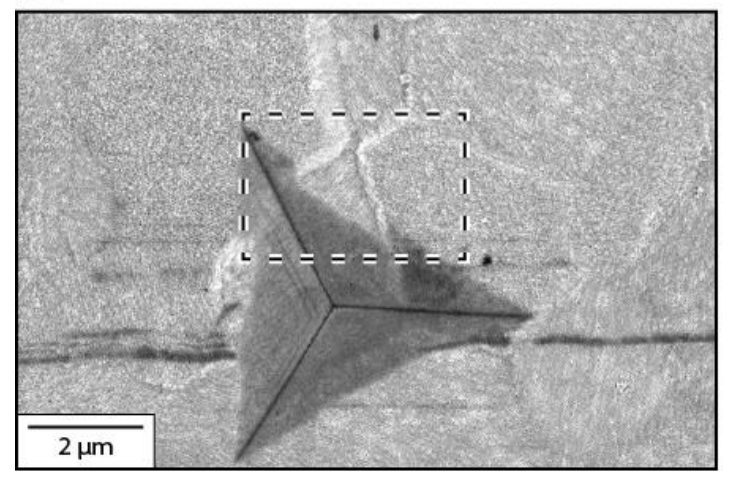

(b)

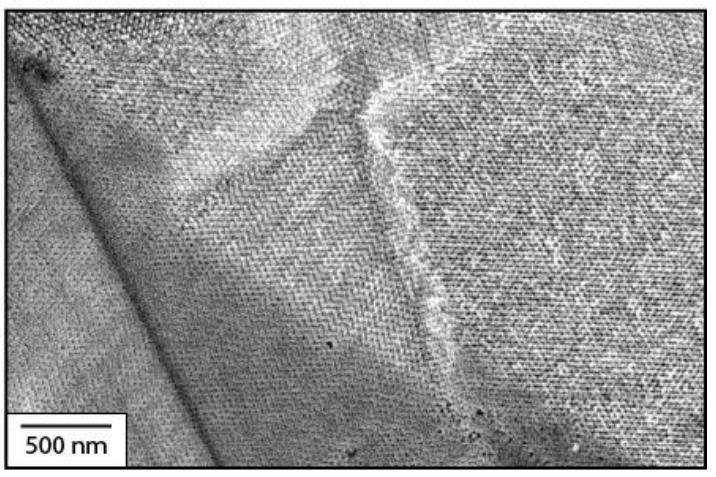

Figure 5: SEM micrographs showing the top view of the double gyroid film indented to a normalised depth $\delta / h \approx 0.73$ by the Berkovich indenter. (a) Micrograph showing the full indented zone and (b) a magnified view of the marked region in (a) near a grain boundary.

\subsection{Measured properties of the Ni gyroid films}

An SEM micrograph of the top indented top surface of the gyroid film is included in Fig. 5 at a normalised indentation depth $\delta / h \approx 0.73$. The indented region is seen to span across multiple domains/grains of the gyroid film. This is typical for most of the indents performed in this study and thus the results presented subsequently should be viewed as averages over multiple gyroid orientations. The measured values of $E_{R}$ and $H$ are plotted in Figs. 6a and 6b, respectively as a function of the normalised indentation depth $\delta / h$. Results are presented showing the mean over all 24 tests conducted on the 4 different gyroid films and error bars showing the standard deviation about this mean are also included ${ }^{1}$. The hardness $H$ is seen to be reasonably independent of $\delta$ especially for $\delta / h \geq 0.2$. At $\delta / h=0.2$, the contact depth $\delta=$ $200 \mathrm{~nm}$ and from Eq. (2.1) the contact area $A_{c} \approx 1.13 \mu \mathrm{m}^{2}$ corresponding to a contact radius $\sqrt{A_{c} / \pi}=600 \mathrm{~nm}$. Thus, for $\delta / h \geq 0.2$ the indentation depth $\delta \gg R_{a}$ and the contact radius is much larger than the gyroid lattice cell size. This results in a measured hardness $H$ that is independent of the indentation depth. By contrast, the reduced modulus $E_{R}$ shows a clear rising trend with increasing $\delta$ presumably due to interactions with the stiff substrate over the whole range of indentation depths investigated here.

The hardness measurements can be used to estimate both the compressive strength of the gyroid lattice and the associated strength of the parent $\mathrm{Ni}$ in the struts of the gyroid lattice. For highly porous (and hence compressible) solids, the indentation hardness is approximately equal to their uniaxial compressive strength [26], i.e. $H \approx$ $\sigma_{Y}^{D G}$, where $\sigma_{Y}^{D G}$ is the uniaxial compressive strength of the double gyroid. We thus estimate that the compressive strength of the $\bar{\rho}^{D G}=0.38 \mathrm{Ni}$ double gyroid lattice to be $\sigma_{Y}^{D G} \approx 0.6 \mathrm{GPa}$. Gibson and Ashby [3] estimate the strength of low relative density isotropic bending-dominated lattices made from parent materials of strength $\sigma_{Y S}$ as

\footnotetext{
1 The variation in the experiments could be partly related to the indentation across differently oriented grains. However, we shall see in Section 3 that the mechanical properties of gyroids are reasonably isotropic and thus the grain structure of the films is not thought to be the primary cause of the observed scatter. The scatter is primarily related to variations between samples: the precise source of this scatter remains a topic for further investigations.
} 
$\sigma_{Y}=0.3 \bar{\rho}^{1.5} \sigma_{Y S}$. Then with, $H \approx \sigma_{Y}^{D G}$ we get the relation between the measured hardness and relative density $\bar{\rho}$ of the single gyroid as

$$
H=2 \times 0.3 \bar{\rho}^{1.5} \sigma_{Y S},
$$

where we have included a factor of 2 in the pre-factor to account for the fact that the measured hardness is for the double gyroid comprising two interpenetrating but independent single gyroids of relative density $\bar{\rho}$. With $H=0.6 \mathrm{GPa}$ and $\bar{\rho}=0.19$, we deduce $\sigma_{Y S}=12.1 \mathrm{GPa}$. This is many times higher than the bulk yield strength of annealed $\mathrm{Ni}$ and in fact higher than usual estimates of the theoretical strength $G_{S} / 10$, where $G_{S}$ is the shear modulus of solid Ni. Thus, while it is conceivable that this approximate analysis overestimates $\sigma_{Y S}$ it nevertheless suggests an anomalously strong parent material response. We attribute this high strength to the "size effect" of the yield strength as reported in numerous recent measurements on the compressive response of $\mathrm{Ni}$ and other metallic micro-pillars [17, 18]. These studies have shown that the compressive strength of micro-pillars increases sharply with decreasing pillar diameter for diameters less than about $0.5 \mu \mathrm{m}$ due to the so-called dislocation starvation phenomenon. In such small pillars, dislocations readily exit from the free surfaces leaving behind dislocation-free specimens that can approach their ideal strength. For example, Dou and Derby [27] proposed that the strength $\sigma_{Y S}$ scales with pillar diameter $d$ for FCC materials such as $\mathrm{Ni}$ and $\mathrm{Au}$ via the relation

$$
\begin{aligned}
& \sigma_{Y S} \\
& =\left\{\begin{array}{cc}
\frac{G_{s}}{10} & d<54.52 b \\
1.4 G_{S}(d / b)^{-0.66}, & 54.52 b \leq d<\left(\frac{1.4 G_{s}}{\sigma_{0}}\right)^{1.52} \\
\sigma_{0} & \text { otherwise, }
\end{array}\right.
\end{aligned}
$$

where $b$ is the Burger's vector and $\sigma_{0}$ the bulk yield strength of the metal. With $b \approx$ $0.25 \mathrm{~nm}$ for $\mathrm{Ni}$, Eq. (2.5) predicts that $\mathrm{Ni}$ struts attain their theoretical strength for $d<13 \mathrm{~nm}$ which is approximately the strut diameters of the double gyroids tested here. This size effect of the strength of the parent material is what results in the anomalously high strengths of the gyroids investigated here. Such observations of ultra-strong micro lattice materials (cell size $\sim 6 \mu \mathrm{m}$ ) made by $3 \mathrm{D}$ printing using a nano-scribe have recently been reported [9] though in those studies the parent materials strength did not approach the theoretical strength of the materials. The parent material strength of the gyroid inferred here is significantly higher than those reported in [9] due to the fact that the gyroids made by the block copolymer route have significantly smaller cell sizes.

The scaling analysis reported here is very approximate and we proceed in Section 3 to report detailed numerical simulations to more accurately estimate the properties of the parent material of the $\mathrm{Ni}$ double gyroids from the indentation measurements reported here. 
(a)

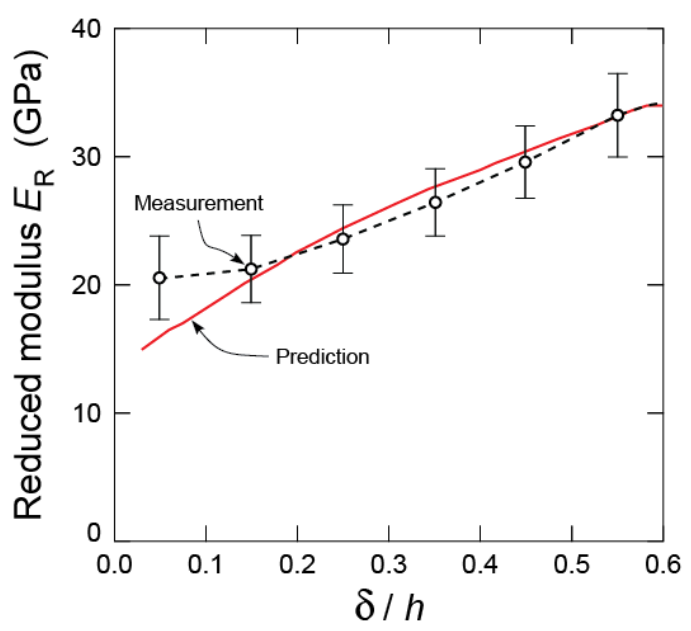

(b)

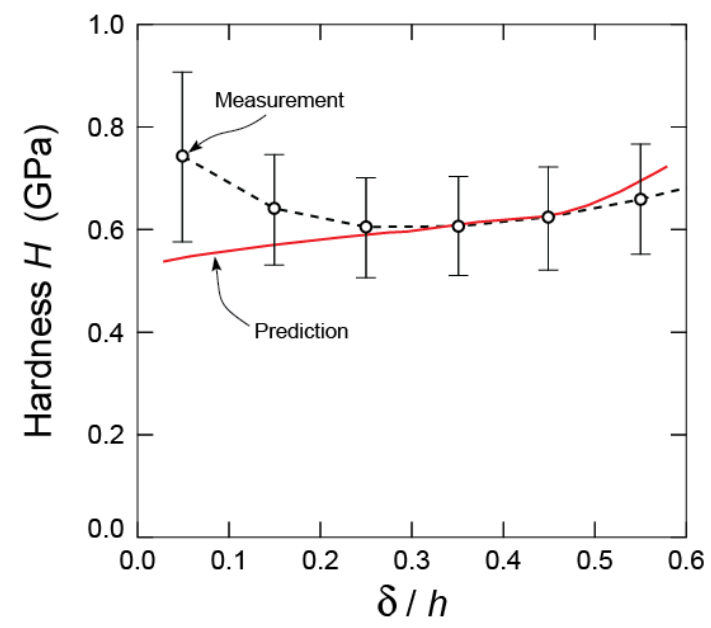

Figure 6: The measured (a) reduced indentation modulus $E_{R}$ and (b) hardness $H$ of the Ni double gyroid films a function of the indentation depth $\delta$ normalised by the double gyroid film thickness $h$. The error bars indicate the standard deviation of the measurements over the 24 tests conducted on 4 different gyroid films. The FE predictions with parent Ni properties $E_{S}=242 \mathrm{GPa}, v_{S}=0.3$ and $\sigma_{Y S}=5.74 \mathrm{GPa}$ are included and seen to be in good agreement with the measurements.

\section{Numerical simulations of the indentation of double gyroid films}

The main aim of this study was to measure the fundamental mechanical properties of the nano double gyroid lattices such as the Young's modulus and uniaxial compressive strength. The indentation measurements reported above do not directly provide these properties. Here we report a multi-scale analysis to extract both the effective mechanical properties of the double gyroids and the properties of the parent material within the gyroid struts from the indentation measurements of Section 2.

The basic outline of the procedure is described here and details provided in subsequent sections. First the double gyroid unit cell is modelled explicitly and threedimensional (3D) finite element (FE) calculations performed to determine the effective properties (Young's modulus, yield strength etc.) of the double gyroid for an assumed set of parent material properties. These effective properties are then used to calibrate a continuum crushable foam model that represents the smeared-out double gyroid lattice. Finite element calculations are then used to determine the indentation response of the gyroid film with the film modelled as a crushable foam and the glass slide substrate as a linear elastic medium. The predicted indentation response is compared against the measurements reported in Section 2. This procedure is iteratively repeated by changing the assumed parent material properties of the gyroids until good agreement of the predicted and measured indentation response is achieved. This converged simulation gives the effective properties of the $\mathrm{Ni}$ double gyroids tested in this study as well as the associated properties of the parent Ni.

\subsection{Effective properties of the double gyroid}

In order to compute the effective properties of the double gyroid we first need to construct a periodic unit cell. A sketch of this unit cell is shown in Fig. 2b. This unit cell is constructed using the approximation to the single gyroid morphology as 
proposed by Lambert et al. [28] and Wohlgemuth et al. [29]. These authors suggested that the surface of a single gyroid is well represented by a function $F-t_{0}=0$, where

$$
\begin{aligned}
F \equiv \sin \left(\frac{2 \pi x}{a}\right) & \cos \left(\frac{2 \pi y}{a}\right) \\
& +\sin \left(\frac{2 \pi y}{a}\right) \cos \left(\frac{2 \pi z}{a}\right)+\sin \left(\frac{2 \pi z}{a}\right) \cos \left(\frac{2 \pi x}{a}\right) .
\end{aligned}
$$

Here $a$ is the periodicity of lattice and $(x, y, z)$ are Cartersian coordinates that are aligned with the cubic directions of the gyroid lattice as shown in Fig. $2 b$ while the scaling parameter $t_{0}$ sets the relative density of the single gyroid. Equation (3.1) results in a gyroid with a connectivity of three struts per node with Plateau border like features near the nodes. The cross-section of the struts of the gyroid changes from an elliptical shape near the nodes to a circular shape at mid-span. The thinning of the struts towards the mid-span implies that the value of $t_{0}$ is limited to $\left|t_{0}\right|<1.41$ as the area of the circular cross section at mid-span vanishes for larger values of $\left|t_{0}\right|$. The double gyroid is then constructed infilling the spaces $F-t_{0} \geq 0$ and $F+t_{0} \leq 0$ to get two interpenetrating single gyroids of opposite chirality as shown in Fig. 2. A value of $\left|t_{0}\right|=0.93$ gives a single gyroid with relative density $\bar{\rho}=0.19$ and corresponding $38 \%$ relative density double gyroid.

The double gyroid geometry was constructed as described above and FE calculations performed to determine the effective elastic and plastic properties of the double gyroid using the commercial finite element package ABAQUS. The double gyroid geometry was meshed using uniform four-noded linear tetrahedral elements (C3D4 in the ABAQUS notation) such that at-least 20 elements were present across the midspan section of the gyroid struts. The parent material of the gyroid was assumed to be an isotropic elastic perfectly-plastic J2 flow theory solid with an elastic Young's modulus $E_{S}$, Poisson's ratio $v_{S}=0.3$ and uniaxial yield strength $\sigma_{Y S}$. Simulations with periodic boundary conditions imposed on the unit cell sketched in Fig. 2b were conducted to determine the three independent elastic constants of the double gyroid (cubic symmetry of the double gyroid implies there are three independent elastic constants) as well as the strength $\sigma_{Y}^{D G}$ and associated plastic Poisson's ratio $v_{p}^{D G}$ for uniaxial compression/tension along one of the cubic directions of the double gyroid (all the three cubic directions are identical).

With $(x, y, z)$ representing the cubic directions of the double gyroid as shown in Fig. 2b, the effective elastic response can be written using Voigt notation as 


$$
\begin{aligned}
& \left(\begin{array}{c}
\varepsilon_{x x} \\
\varepsilon_{y y} \\
\varepsilon_{z z} \\
2 \varepsilon_{y z} \\
2 \varepsilon_{x z} \\
2 \varepsilon_{x y}
\end{array}\right) \\
& =\left(\begin{array}{cccccc}
1 / E^{D G} & -v^{D G} / E^{D G} & -v^{D G} / E^{D G} & 0 & 0 & 0 \\
-v^{D G} / E^{D G} & 1 / E^{D G} & -v^{D G} / E^{D G} & 0 & 0 & 0 \\
-v^{D G} / E^{D G} & -v^{D G} / E^{D G} & 1 / E^{D G} & 0 & 0 & 0 \\
0 & 0 & 0 & 1 / G^{D G} & 0 & 0 \\
0 & 0 & 0 & 0 & 1 / G^{D G} & 0 \\
0 & 0 & 0 & 0 & 0 & 1 / G^{D G}
\end{array}\right)\left(\begin{array}{c}
\sigma_{x x} \\
\sigma_{y y} \\
\sigma_{z z} \\
\sigma_{y z} \\
\sigma_{x z} \\
\sigma_{x y}
\end{array}\right),
\end{aligned}
$$

where $E^{D G}$ is the Young's modulus, $G^{D G}$ the shear modulus and $v^{D G}$ the Poisson's ratio. The predicted variations of these elastic constants with the relative density $\bar{\rho}^{D G}=2 \bar{\rho}$ are plotted in Fig. 7a $(\bar{\rho}$ varied in the FE calculations by changing the gyroid geometry via the scaling parameter $t_{0}$ ). The scaling of $E^{D G}$ and $G^{D G}$ with $\bar{\rho}^{D G}$ is approximately quadratic: the gyroid lattice has a connectivity of three struts per node and consequently is a bending-dominated structure [5] that gives this quadratic scaling.

(a)

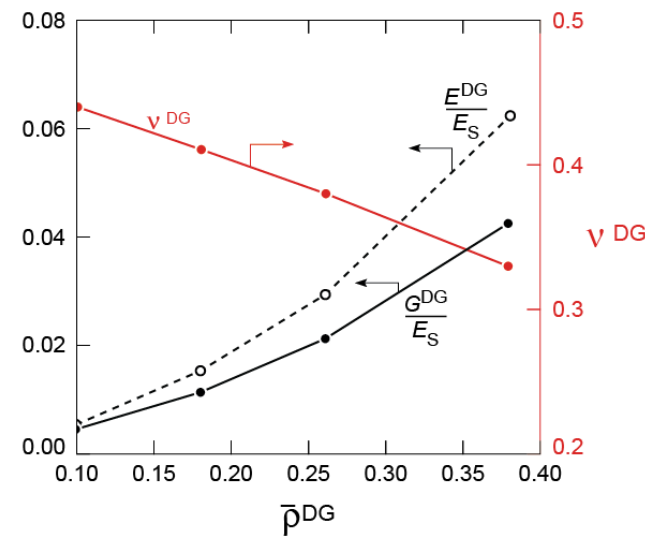

(b)

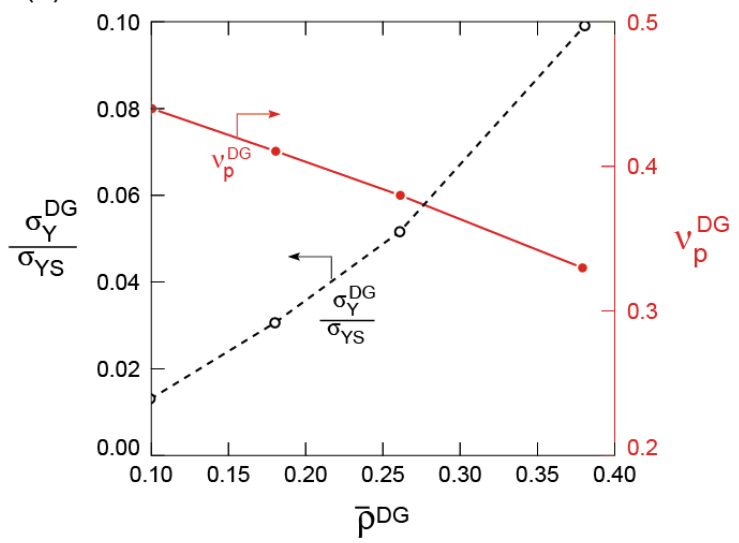

Figure 7: FE predictions of the variation of the mechanical properties of a double gyroid made from an isotropic elastic perfectly plastic material. The variation with relative density $\bar{\rho}^{D G}$ of (a) the normalized elastic moduli $E^{D G} / E_{S}, G^{D G} / E_{S}, v^{D G}$ and (b) the normalized uniaxial strength $\sigma_{Y}^{D G} / \sigma_{Y S}$ along a cubic direction as well as the corresponding plastic Poisson's ratio $v_{p}^{D G}$.

Next consider the strength predictions of the double gyroid lattice. Either elastic buckling or plastic yielding of the struts sets the strength of the gyroid with elastic buckling expected to be the operative collapse mode at combinations of high parent material yield strain $\varepsilon_{Y S} \equiv \sigma_{Y S} / E_{S}$ and low relative density $\bar{\rho}^{D G}$ [3]. The calculations presented here were conducted with a parent material yield strain $\varepsilon_{Y S}=0.03$ and elastic buckling was not observed to be operative over the range $\bar{\rho}^{D G} \geq 0.1$ 
investigated here. Thus, the strength calculations presented here are valid for double gyroids made from parent materials with $\varepsilon_{Y S} \leq 0.03$. Predictions of the variation of the uniaxial compressive/tensile strength $\sigma_{Y}^{D G}$ with $\bar{\rho}^{D G}$ are included in Fig. $7 \mathrm{~b}$ : intriguingly $\sigma_{Y}^{D G}$ scales nearly linearly with $\bar{\rho}^{D G}$. Scaling arguments based on modelling the gyroids struts as slender beams suggest $\sigma_{Y}^{D G} \propto\left(\bar{\rho}^{D G}\right)^{1.5}$ as discussed in Section 2.3. However, the slender beam assumption is not appropriate for double gyroids with $\bar{\rho}^{D G} \geq 0.1$ and the full $3 \mathrm{D}$ FE calculations presented here suggest that double gyroids have a strength that scales nearly linearly with relative density over the range investigated here. Double gyroids are compressible cellular solids and the plastic Poisson's ratio is another property typically considered relevant to characterising the plastic response of such materials. For uniaxial stressing in the $x$-direction, the plastic Poisson's ratio $v_{p}^{D G}$ is defined in terms of the plastic strain increment rates $\dot{\varepsilon}_{x x}^{p}$ and $\dot{\varepsilon}_{y y}^{p}=\dot{\varepsilon}_{z z}^{p}$ as $v_{p}^{D G} \equiv-\dot{\varepsilon}_{y y}^{p} / \dot{\varepsilon}_{x x}^{p}$. Predictions of the variation of $v_{p}^{D G}$ with $\bar{\rho}^{D G}$ are included in Fig. $7 \mathrm{~b}$ : unlike most high porosity cellular solids these double gyroids have a relative high $v_{p}^{D G}$ that ranges between 0.45 and 0.35 for the $\bar{\rho}^{D G}$ values investigated here. Intriguingly, $v_{p}^{D G}$ decreases with increasing $\bar{\rho}^{D G}$ (though in the fully dense limit of $\bar{\rho}^{D G}=1$ the incompressible limit of the parent material is expected to be recovered). This is thought to be related to the details of the gyroid morphology and chirality, which results in a self-folding collapse mode.

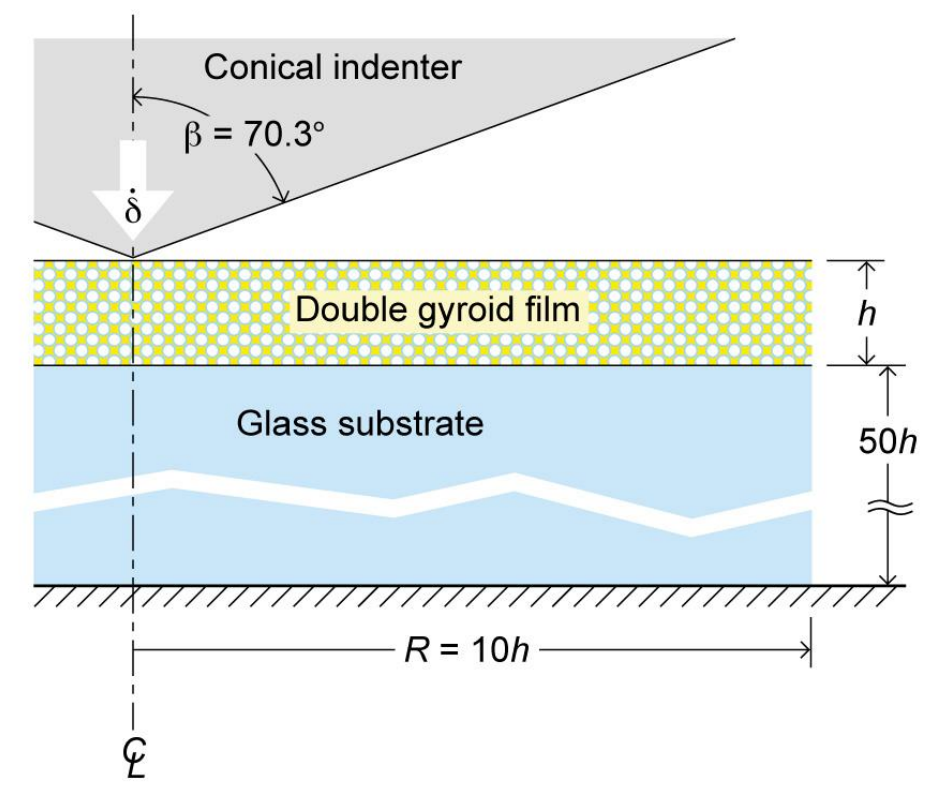

Figure 8: Sketch of axisymmetric conical indentation model used to predict the Berkovich nano indentation response of gyroid films on a glass substrate. The dimensions of the different layers are indicated in terms of the gyroid film thickness $h$.

\subsection{Indentation response of double gyroid films}

In the experiments reported in Section 2.3, the indenter contact radii were significantly larger than the gyroid cell sizes over the majority of the range of indentation depths investigated. Thus, a large number of gyroid cells are being deformed during the indentation process with the wavelengths associated with the deformation field large compared to the gyroid cell size. Thus, the discreteness of the gyroid microstructure is expected to play a negligible role in the indentation response 
detailed in Section 2.3 and it is reasonable to model the gyroid lattice by an appropriate smeared-out continuum.

The gyroid lattices are crushable cellular solids with cubic symmetry. The multi-axial collapse surface calculations of Khaderi et al. [23] show that both the elastic and plastic properties of the gyroids lattice are reasonably isotropic. We thus model the double gyroids lattices using the isotropic crushable foam model of Deshpande and Fleck [30]. This essentially implies that we are modelling indentation over multiple grains (as per the experiments) with the parameters of the Deshpande and Fleck [30] constitutive model representing and an ensemble average of the gyroid properties over multiple orientations. Here we briefly describe this constitutive model and detail its calibration to simulate the double gyroids films. Write $s_{i j}$ as the deviatoric stress and the von-Mises effective stress as $\sigma_{e} \equiv \sqrt{(3 / 2) s_{i j} s_{i j}}$. The isotropic yield surface of the double gyroid lattice is then specified by

$$
\hat{\sigma}-Y\left(\hat{\varepsilon}^{p}\right)=0
$$

where the equivalent stress $\hat{\sigma}$ is a homogenous function of $\sigma_{e}$ and the mean stress $\sigma_{m} \equiv \sigma_{k k} / 3$ according to

$$
\hat{\sigma}^{2} \equiv \frac{1}{1+\left(\frac{\alpha}{3}\right)^{2}}\left[\sigma_{e}^{2}+\alpha^{2} \sigma_{m}^{2}\right]
$$

and $\hat{\varepsilon}^{p}$ the plastic strain work-conjugate to $\hat{\sigma}$. Moreover, $Y\left(\hat{\varepsilon}^{p}\right)$ is the uniaxial yield strength that is a function of the $\hat{\varepsilon}^{p}$ and the parameter $\alpha$ denotes the ratio of deviatoric to hydrostatic strength. Note that the normalisation factor on the right-hand side of Eq. (3.4) is chosen such that $\hat{\sigma}$ denotes the stress in a uniaxial tension or compression test. Normality of plastic flow is assumed and this implies that the plastic Poisson's ratio $v_{p}^{D G}$ is related to $\alpha$ via

$$
v_{p}^{D G}=\frac{1 / 2-(\alpha / 3)^{2}}{1+(\alpha / 3)^{2}}
$$

The double gyroids lattice is expected to behave like a cellular solid with its uniaxial compressive response characterised by a plateau strength $\sigma_{Y}^{D G}$ followed by densification due to contact between the cell walls. This densification strain is reasonably independent of the relative density for periodic lattices [31] and thus we assume $Y\left(\hat{\varepsilon}^{p}\right)$ to have the form

$$
Y= \begin{cases}\sigma_{Y}^{D G} & \hat{\varepsilon}^{p} \leq 0.6 \\ \sigma_{Y}^{D G}+h_{p}\left(\hat{\varepsilon}^{p}-0.6\right) & \text { otherwise }\end{cases}
$$


where the hardening rate $h_{p}$ beyond densification is assumed to be equal to the Young's modulus $E^{D G}$ of the double gyroid. The total strain increment is given by the sum of the elastic and plastic strain increments and here we assume an isotropic elastic response with Young's modulus $E^{D G}$ and Poisson's ratio $v^{D G}$.

Indentation calculations are reported here for a double gyroid of relative density $\bar{\rho}^{D G}=0.38$ made from a parent material with Young's modulus $E_{S}$, Poisson's ratio $v_{S}=0.3$ and yield strength $\sigma_{Y S}$. The calculations of Section 3.1 then specify $v_{p}^{D G} \approx$ $v^{D G} \approx 0.35$ and it follows from Eq. (3.5) that $\alpha=1$. Moreover, for $\bar{\rho}^{D G}=0.38$ we see from Fig. 7 that $\sigma_{Y}^{D G}=0.1 \sigma_{Y S}$ and $E^{D G}=0.062 E_{S}$. The parent material modulus $E_{S}$ and strength $\sigma_{Y S}$ are therefore treated as unknown parameters and varied in order to bring the predicted and measured indentation responses into good agreement.
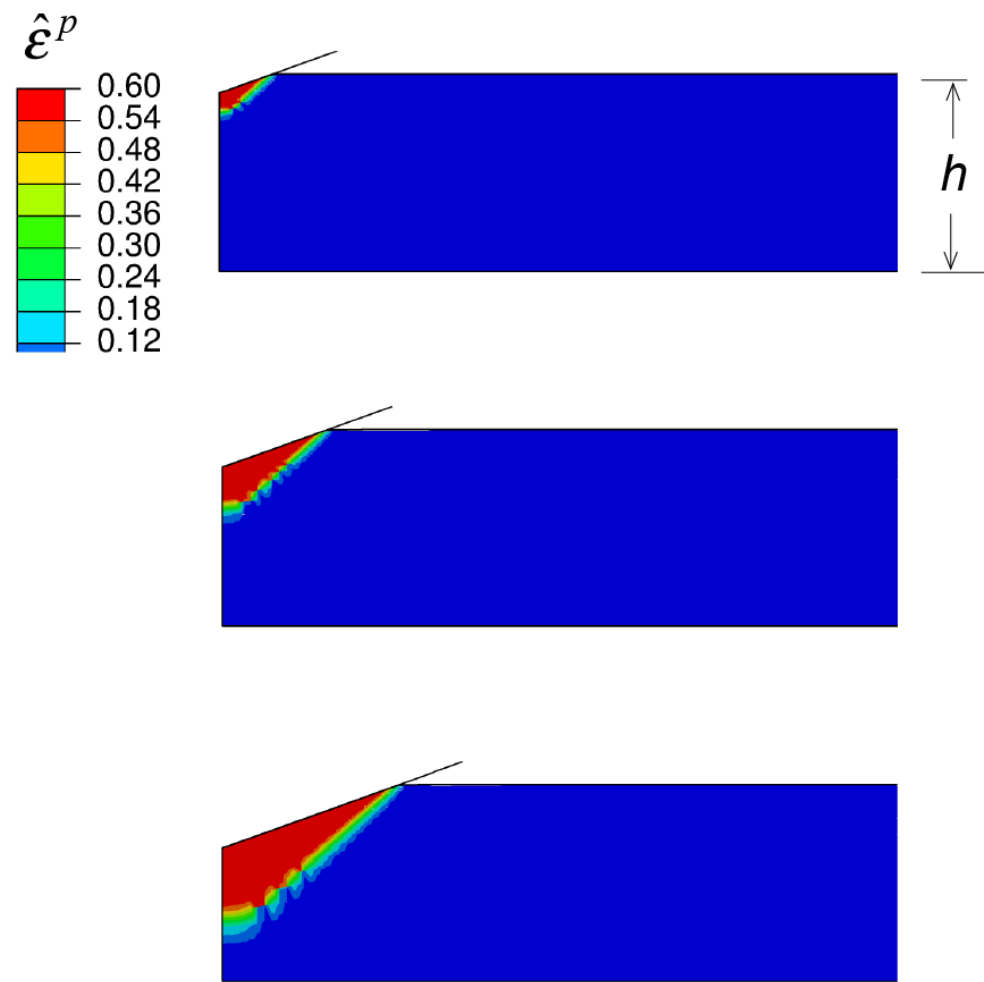

Figure 9: FE predictions of the distribution of the effective plastic strain $\hat{\varepsilon}^{p}$ immediately under the conical indenter at three selected values of the normalized indentation depth $\delta / h=$ $0.1,0.2$ and 0.3 .

The indentation experiments on the double gyroid films were performed using a Berkovich indenter. Numerous studies including 3D FE calculations [32] have shown that it suffices to model the Berkovich indenter by a conical indenter with semi-angle $\beta=70.3^{\circ}$ as shown in Fig. 8 so that the nominal contact area $A_{c}$ versus indentation depth $\delta$ relation for this equivalent conical is equal to that of the ideal Berkovich indenter. Thus, we employ a simplified axisymmetric model (Fig. 8) for the indentation of the double gyroid films on glass substrates of thicknesses $h$ and $50 h$, respectively and radius $R=10 \mathrm{~h}$. The glass substrate was modeled as linear elastic solid with modulus $69.6 \mathrm{GPa}$ and Poisson's ratio 0.3 while the conical indenter was assigned the properties of diamond, i.e. again a linear elastic solid with modulus $E^{I}=$ 
$1140 \mathrm{GPa}$ and Poisson's ratio $v^{I}=0.07$. The double gyroid film was assumed to be perfectly bonded to the substrate with material properties as detailed above. Quasistatic finite strain indentation calculations were performed with contact between the indenter surface and gyroid film modelled using the Master-Slave surface contact algorithm in ABAQUS. In line with the experimental protocol, successive loading and unloading cycles were performed to measure the applied load $P$ versus indentation depth $\delta$ response and the reduced modulus $E_{R}$ and hardness $H$ extracted as in the experiments; i.e. using the Oliver and Pharr [22] method. In the case of these FE calculations, this implies that the contact area versus depth relation is given by $A_{c}=$ $\pi \delta^{2} \tan ^{2} \beta \approx 24.5 \delta^{2}$ and the hardness $H=P(\delta) / A_{c}(\delta)$ at a depth $\delta$. The reduced modulus is also inferred by fitting a power law to the unloading curve and then using the Sneddon formula as described in Section 2.1.

FE predictions of the reduced modulus $E_{R}$ and hardness $H$ as a function of the normalized indentation depth $\delta / h$ are included in Figs. 6a and 6b, respectively for parent material properties $E_{S}=242 \mathrm{GPa}$ and $\sigma_{Y S}=5.74 \mathrm{GPa}$ (i.e. $\left.\varepsilon_{Y S}=0.023\right)^{2}$. These values of the parent material properties bring the measured and predicted indentation responses into good agreement (to within the scatter of the experimental data) over the whole range of $\delta$ investigated here. This then implies that the $\bar{\rho}^{D G}=$ $0.38 \mathrm{Ni}$ double gyroids investigated here have a Young's modulus and uniaxial compressive strength of $E^{D G}=15 \mathrm{GPa}$ and $\sigma_{Y}^{D G}=574 \mathrm{MPa}$, respectively. We note in passing that the stresses $\sigma_{\gamma}$ generated in the gyroid struts due to surface energy are on the order of $100 \mathrm{MPa}\left(\sigma_{\gamma} \sim \gamma / d\right.$, where $\gamma=1 \mathrm{~J} \mathrm{~m}^{-2}$ is the surface energy and $d=$ $10 \mathrm{~nm}$ the diameter of the gyroid strut) and is thus small compared to the estimated material strength of $\sigma_{Y S}=5.74 \mathrm{GPa}$. The continuum modelling approach presented here is therefore sufficient without the need to explicit account for surface effects via lower length scale molecular dynamics calculations.

Thus, consistent with the approximate estimates presented in Section 2.3, we find that the Young's modulus of the parent solid material is in line with that that of bulk Ni. However, the strength $\sigma_{Y S}$ is at-least a factor of 20 higher compared to bulk annealed $\mathrm{Ni}$ and on the order of usual estimates of the theoretical strength $G_{S} / 10$. This anomalously strong parent material response is due to the lack of dislocations within the very small diameter gyroid struts as discussed in Section 2.3.

Contour plots of the equivalent plastic strain $\hat{\varepsilon}^{p}$ immediately underneath the indenter at three selected values of the normalized indentation depth $\delta / h$ are included in Fig. 9. Consistent with numerous studies in the literature on the indentation of porous solids [26], we observe that the material immediately in contact with the indenter has fully densified with active plastic straining taking place in a small intermediate zone between the densified region and the surrounding elastic material. The deformation proceeds in approximately a self-similar manner with the densified zone increasing in size. Importantly we observe that there is negligible pile-up or sink-in around the edges of the indented zone consistent with the assumptions made in the extraction of $E_{R}$ and $H$ from the indentation measurements.

\footnotetext{
2 Recall that the FE calculations of Fig. $7 \mathrm{~b}$ are valid for $\varepsilon_{Y S} \leq 0.03$ and thus the parent material parameters inferred here lie within this range of validity.
} 


\section{Nickel nano-gyroids in strength versus density material space}

We have estimated the Young's modulus and strength of the $\bar{\rho}^{D G}=0.38 \mathrm{Ni}$ double gyroid to be $E^{D G}=15 \mathrm{GPa}$ and $\sigma_{Y}^{D G}=574 \mathrm{MPa}$, respectively. Thus, porous Ni with $\sim 60 \%$ porosity has a compressive strength that is higher than that of the strongest bulk Ni alloys even though this double gyroid lattice material is approximately 2.5 times lighter compared to bulk $\mathrm{Ni}$. Consequently, the measured $\mathrm{Ni}$ nano gyroid strength apparently violates the non-linear Hashin-Shtrikman bound as seen in Fig. 1. This is due to the fact that nano size struts have an anomalously high strength $\sigma_{Y S}=$ $5.74 \mathrm{GPa}$ which is much higher than that of bulk Ni.

There is a growing literature on ultra-strong micro lattices and thus it is instructive to compare the strength of the Ni double gyroid lattices with other materials including the recently manufactured nano/micro lattice materials. The double gyroid strength predictions of Fig. $7 \mathrm{~b}$ are plotted in the Ashby chart in Fig. 1 using an assumed parent $\mathrm{Ni}$ strength $\sigma_{Y S}=5.74 \mathrm{GPa}$ and density of the solid Ni $\rho_{S}=8900 \mathrm{~kg} \mathrm{~m}^{-3}$. The measured strength of the $\bar{\rho}^{D G}=0.38$ double gyroid lattice (which has a density $\rho=$ $\bar{\rho}^{D G} \rho_{S}=3380 \mathrm{~kg} \mathrm{~m}^{-3}$ ) is explicitly marked in the Fig. 1 and lies above even the strongest solid Ni alloys. A similar observation of a metallic porous lattice material having a strength higher than the equivalent bulk material has been previously reported for micron scale $\mathrm{Cu}$ octet truss lattice materials built via micro 3D printing [9] and these measurements are included in Fig. 1. The octet truss is a stretching governed and generally considered a practical optimal topology in terms of the stiffness and strength of a nearly isotropic lattice material. Nevertheless as seen in Fig. 1, these octet truss lattices have a lower strength to density ratio compared to the bending-dominated double gyroid lattices. This is due to the fact that the minimum unit cell size of the octet trusses investigated by $\mathrm{Gu}$ and Greer [9] was $6 \mu \mathrm{m}$ and consequently via Eq. (2.5) the parent material was weaker in these micro octet truss lattices compared to parent material of the nano gyroids: the high parent material strength at the nano sizes more than compensates for the loss of strength due to the bending-governed gyroid topology.

The strength of micro/nano-scale prismatic ceramic lattices again made via 3D printing are also included in Fig. 1. In the prismatic directions these essentially twodimensional materials outperform the nano gyroid lattices. However, we emphasize that the gyroids are nearly isotropic while the prismatic lattices are strongly anisotropic with very weak in-plane responses.

\section{Concluding remarks}

We have reported indentation measurements and associated simulations to estimate the mechanical properties of Ni nano double gyroids. Nickel double gyroid films of thickness approximately $1 \mu \mathrm{m}$ with unit cell sizes on the order of $45 \mathrm{~nm}$ and a relative density of $38 \%$ were manufactured by block co-polymer self-assembly followed by electro-deposition of the $\mathrm{Ni}$. Berkovich nano indentation tests were performed to determine both the hardness and modulus of the films. The measurements revealed a high hardness of $\sim 0.6 \mathrm{GPa}$ and no discernable indentation size effect on the hardness measurements.

A multi-scale finite element analysis was performed to extract both the effective mechanical properties of the gyroids and the associated properties of the parent $\mathrm{Ni}$. 
This analysis suggested that the uniaxial compressive strength of the double gyroids was approximately equal to its hardness as the material is plastically compressible with negligible indentation constraint effects. The parent $\mathrm{Ni}$ was estimated to have the usual Young's modulus of bulk Ni (i.e. $240 \mathrm{GPa}$ ) but its yield strength was predicted to be about $5.7 \mathrm{GPa}$, i.e. approaching the theoretical strength value of $\mathrm{Ni}$. This anomalously high strength was due to the fact that the gyroid struts had a diameter of approximately $13 \mathrm{~nm}$ and were thus essentially dislocation free. As a consequence, the compressive strength of $\mathrm{Ni}$ nano double gyroids was approximately equal to that of fully dense high strength $\mathrm{Ni}$ alloys even though the Ni double gyroids comprised approximately $60 \%$ porosity.

The self-assembly process employed here enables the relatively fast and easy manufacture of nano double gyroid lattices. These lattices have a bending-dominated topology, which is non-optimal from a mechanical property viewpoint. However, selfassembly enables very small cell sizes to be readily achieved and here we have demonstrated that the plasticity size effect at these small length scales more than compensates for the non-optimal topology. In fact, Ni nano double gyroids have a higher strength to weight ratio compared to $\mathrm{Cu}$ micro lattices with the near optimal octet truss topology.

\section{Acknowledgements}

We are grateful to the DARPA MCMA program (Grant Number W91CRB-10-1-005) for the financial support of this work. The program was overseen by Dr Judah Goldwasser of DARPA. The authors are also grateful for financial support in the form of an ERC MULTILAT grant 669764. US acknowledges funding from the Adolphe Merkle Foundation and from the Swiss National Science Foundation. MRJS acknowledges support from the Nokia Research Centre Cambridge.

\section{References}

[1] M. F. Ashby (2005), Materials Selection in Mechanical Design, Elsevier Ltd.

[2] N.A. Fleck, V.S. Deshpande and M.F. Ashby (2010), Micro-architectured materials: past, present and future, Proceedings of the Royal Society A, 466:24952516.

[3] L.J. Gibson and M.F. Ashby (1999), Cellular Solids: Structure and Properties, second ed., Cambridge University Press.

[4] V.S. Deshpande, N.A. Fleck and M.F. Ashby (2001), Effective properties of the octet-truss lattice material, Journal of the Mechanics and Physics of Solids, 49:1747-1769.

[5] V. S. Deshpande, M. F. Ashby and N. A. Fleck (2001), Foam topology bending versus stretching dominated architectures, Acta Materialia, 49:1035-1040.

[6] H.N.G. Wadley, N.A. Fleck and A.G. Evans (2003), Fabrication and structural performance of periodic cellular metal sandwich structures, Composite Science and Technology, 63:2331-2343.

[7] Y. Chunze, L. Hao, A. Hussein, P. Young, J. Huang and W. Zhu (2015), Microstructure and mechanical properties of aluminium alloy cellular lattice structures manufactured by direct metal laser sintering, Materials Science \& Engineering A, 628:238-246.

[8] G.W Kooistra, V.S. Deshpande and H.N.G. Wadley (2004), Compressive 
behaviour of age hardenable tetrahedral lattice truss structures made from aluminium, Acta Materialia, 52:4229-4237.

[9] X.W. Gu and J. R. Greer (2015), Ultra-strong architected Cu meso-lattices, Extreme Mechanics Letters, 2:7-14.

[10] T.A. Schaedler, A.J. Jacobsen, A. Torrents, A.E. Sorensen, J. Lian, J.R. Greer, L. Valdevit and W.B. Carter (2011), Ultralight Metallic Microlattices, Science, 334:962-965.

[11] X. Zheng, H. Lee, T.H. Weisgraber, M. Shusteff, J. Deotte, E.B. Duoss, J.D. Kuntz, M.M. Biener, Q. Ge, J.A. Jackson, S.O. Kucheyev, N.X. Fang and C.M. Spadaccini (2014), Ultralight, ultrastiff mechanical metamaterials, Science, 344:13731377.

[12] J. Bauer, S. Hengsbach, I. Tesari, R. Schwaiger and O. Kraft (2014), Highstrength cellular ceramic composites with 3D microarchitecture, Proceedings of the National Academy of Sciences of the United States of America, 111:2453-2458.

[13] P. M. Suquet (1993), Overall potentials and extremal surfaces of power law or ideally plastic composites, Journal of the Mechanics and Physics of Solids, 41:9811002.

[14] L. Dong, V.S. Deshpande and H.N.G. Wadley (2015), Mechanical performance of Ti-6Al-4V Octet-Truss Lattice Structures, International Journal of Solids and Structures, 60:107-124.

[15] N.A. Fleck, G.M. Muller, M.F. Ashby and J.W. Hutchinson (1994), Strain gradient plasticity: theory and experiment. Acta Metallurgica et Materialia, 42:475487.

[16] W. D. Nix and H. Gao (1998), Indentation size effects in crystalline materials: a law for strain gradient plasticity, Journal of the Mechanics and Physics of Solids, 46:411-425.

[17] M.D. Uchic, P.A. Shade and D.M. Dimiduk (2009), Plasticity of micrometerscale single crystals in compression, Annual Review of Materials Research, 39:361386.

[18] J.R. Greer and J.T.M. De Hosson (2011), Plasticity in small-sized metallic systems: intrinsic versus extrinsic size effect, Progress in Materials Science, 56:654724.

[19] D.C. Jang, L.R. Meza, F. Greer and J.R. Greer (2013), Fabrication and deformation of three-dimensional hollow ceramic nanostructures, Nature Materials, 12:893-898.

[20] L.C. Montemayor, L.R. Meza and J.R. Greer (2013), Design and fabrication of hollow rigid nanolattices via two-photon lithography, Advanced Engineering Materials, 16:184-189.

[21] M.R.J. Scherer, L. Li, P.M.S. Cunha, O.A. Scherman and U. Steiner (2012), Enhanced electrochromism in gyroid-structured vanadium pentoxide, Advanced Materials, 24:1217-1221.

[22] W.C. Oliver and G.M. Pharr (1992), An improved technique for determining hardness and elastic modulus using load and displacement sensing indentation experiments, Journal of Materials Research, 7:1564-1582.

[23] S.N. Khaderi, V.S. Deshpande and N.A. Fleck (2014), The stiffness and strength of the gyroid lattice, International Journal of Solids and Structures, 51:38663877.

[24] I.N. Sneddon (1948), Boussinesq's problem for a rigid cone, Proceedings of the Cambridge Philosophical Society, 44: 492-507.

[25] R. Cooper (2007), Ubi 1 Software Version 8.1.1 User Manual, Hysitron Inc. 
[26] N.A. Fleck, H. Otoyo and A. Needleman (1992), Indentation of porous solids. International Journal of Solids and Structures, 26:1613-1636.

[27] R. Dou and B. Derby (2009), A universal scaling law for the strength of metal micropillars and nanowires, Scripta Materialia, 61:524-527.

[28] C.A. Lambert, L.H. Radzilowski and E.L. Thomas (1996), Triply periodic level surfaces as models for cubic tricontinuous block copolymer morphologies, Philosophical Transactions of the Royal Society A, 354:2009-2023.

[29] M. Wohlgemuth, N. Yufa, J. Hoffman and E.L. Thomas (2001), Triply periodic bicontinuous cubic microdomain morphologies by symmetries, Macromolecules, 34:6083-6089.

[30] V.S. Deshpande and N.A. Fleck (2000), Isotropic constitutive models for metallic foams, Journal of the Mechanics and Physics of Solids, 48:1253-1283.

[31] S.M. Pingle, N.A. Fleck, V.S. Deshpande and H.N.G. Wadley (2011), Collapse mechanism maps for a hollow pyramidal lattice, Proceedings of the Royal Society A, 467:985-1011.

[32] M. Li, W.M. Chen, N.G. Liang and L.D. Wang (2004), A numerical study of indentation using indenters of different geometry, Journal of Materials Research, 19:73-78. 